\title{
Cáncer de próstata metastático
}

\section{Resumen}

El cáncer de próstata es una de las neoplasias más frecuentes en los países occidentales. Representa el $29 \%$ de las neoplasias malignas, y el $11 \%$ de las muertes por cáncer en los varones de los Estados Unidos. La cirugía y la radioterapia constituyen el tratamiento estándar de la enfermedad localizada; existiendo tratamientos efectivos para la enfermedad metástatica.

La terapia estándar en la enfermedad metastática es la ablación androgénica por medio quirúrgico o médico. La deprivación androgénica produce una disminución en los niveles de PSA, una regresión de masas tumorales medibles y un período de estabilidad clínica en el que el tumor no sufre variaciones en tamaño, seguido de un período variable de tiempo en el que se incrementa el PSA, reaparece la proliferación del tumor y se produce una progresión de la enfermedad tumoral clínicamente detectable. La primera consideración terapéutica en los pacientes con cáncer de próstata metastático probablemente hormo-refractario, es la demostración de los niveles séricos de testosterona a rango de castración. Si los niveles séricos de testosterona están en rango de castración, existen diversas modalidades terapéuticas, tales como la hormonoterapia de segunda línea, la quimioterapia y los agentes experimentales.

Por primera vez, en 2004, un régimen de quimioterapia basado en docetaxel demostró prolongar la supervivencia de los casos de cáncer de próstata que habían progresado tras la hormonoterapia.

\section{Palabras clave:}

Cáncer de próstata. Docetaxel. Hormonorefractario. LHRH.

Oncología, 2006; 29 (10):398-404

Servicio de Oncología Médica

${ }^{1}$ Jefe de Servicio de Oncología Médica

Hospital General Universitario Gregorio Marañón. Madrid
Recibido: 20.01 .06

Revisado: 06.02 .06

Aceptado: 02.03.06 


\section{Summary}

Prostate cancer is one of the most frequent tumors in the industrialized countries. It accounts for $29 \%$ of male malignancies and $11 \%$ of male cancer deaths in the United States. Surgery and radiation compose the standard management to treat the localized disease, and there are efficacious treatments for the metastatic disease.

The aim of the standard treatment for metastatic prostate cancer is to eliminate the androgenic activity by medical or surgical means. Androgen deprivation produces a diminution of PSA, a regression of measurable tumor mass, and a period of clinical stability without observable tumor size changes, that are followed by the rise of PSA, tumor proliferation and clinically detectable tumor regrowth. The first consideration when facing the adequate treatment of a possible hormone-refractory metastatic prostate cancer is to know if the testosterone level is in the castration range. If this can be asserted, it is necessary to decide between the several available treatment options, including secondline hormonal therapy, chemotherapy, and clinical assay agents.

For the first time, in 2004 a docetaxel-based chemotherapy program proved to prolong the lives of prostate cancer patients who had shown progression on hormone therapy.

Key words: Prostate cancer. Docetaxel. Hormone-refractory. LHRH.

\section{Introducción}

El cáncer de próstata $(\mathrm{CP})$ es una de las neoplasias más frecuentes en el mundo occidental. El CP en España presenta una tasa ajustada de mortalidad del 13,52\%, siendo el cuarto cáncer en incidencia y el tercero en mortalidad en nuestro país ${ }^{1}$. Su incidencia aproximada en España es de 22,2/100.000 habitantes/año y su prevalencia estimada de 200/100.000.

La frecuencia del CP se incrementa con la edad, siendo la edad mediana al diagnóstico de aproximadamente 70 años ${ }^{2}$.

Es importante su clasificación en enfermedad localizada (EL), locorregionalmente avanzada (ELRA) y cáncer de próstata metastático (CPM), desde el punto de vista de la actitud terapéutica y pronostica. En los estadios localizados la intención del tratamiento es radical, mientras que en los estadios avanzados es paliativa.

La supervivencia global de la enfermedad a los 5 años es de un 76-80\% para todos los estadios en global, mientras que para la EL es de $92-95 \%$, para la ELRA de un $80-83 \%$, y en el CPM es de $29 \%{ }^{3}$.

\section{Cáncer de próstata metastático hormonosensible}

El cáncer de próstata es una neoplasia formada por una población celular heterogénea desde el inicio. Las células tumorales de adenocarcinoma coexisten con otras subpoblaciones celulares que presentan diferenciación neuroendorina ${ }^{4}$, de células microcíticas y de carcinoma transicional ${ }^{5}$.

El CPM es muy sensible a la deprivación androgénica en su fase inicial (fase de hormonosensiblidad o andrógeno-dependencia), con una mediana de duración de respuesta al tratamiento de unos 12-18 meses, después de lo cual progresará a una fase de hormonorresistencia o andrógeno-independencia ${ }^{6}$. Con la deprivación andrógenica de primera línea, aproximadamente el $80 \%$ de los casos de CPM responden al tratamiento (respuesta bioquímica, objetiva y sintomática).

Este fenómeno se debe a que las células glandulares prostáticas precisan para su crecimiento y diferenciación del estímulo de los andrógenos (testosterona y su metabolito activo dihidrotestosterona), y en ausencia de los andrógenos dichas células entran en apoptosis. No obstante, hasta un $43 \%$ 
de las muestras examinadas tras la ablación androgénica siguen mostrando áreas tumorales inalteradas, motivo por el cual este tratamiento no es curativo $^{7}$. La supervivencia mediana en los pacientes con CPM desde el inicio de la ablación hormonal es de unos 3 años, y en los casos resistentes de un año.

La deprivación androgénica se puede obtener por distintos métodos, entre los cuales destaca los análogos de LHRH:

1. Castración quirúrgica. Se obtiene mediante la orquiectomía bilateral. Se trata de un procedimiento económico, técnicamente sencillo y seguro, produciendo una ablación irreversible. Sin embargo es mal aceptado por los pacientes por las implicaciones psicológicas que ello supone.

2. Análogos de LHRH. Son compuestos sintéticos análogos de la molécula de LHRH, en los que se sustituye una leucina por una glicina en la posición 6. Estos fármacos producen un estímulo continuado de la hipófisis, lo cual provoca el "agotamiento" de la misma; y de esta manera inhiben la producción de los andrógenos testiculares. Estos fármacos producen un incremento inicial de los niveles séricos de testosterona, lo que puede provocar un fenómeno de "flare" o empeoramiento de la enfermedad, sobre todo en pacientes con compresión medular o uropatía obstructiva. Por este motivo, en las primeras semanas del tratamiento deben administrarse conjuntamente con los antiandrógenos ${ }^{8}$.

Estos fármacos se consideran de primera elección en la primera línea de hormonoterapia en los $\mathrm{CPM}^{8,9}$. Los más usados en la práctica clínica son la goserelina ${ }^{10}(10,8 \mathrm{mg}$ trimestral por vía subcutánea, Zoladex), buserelina $(6,3 \mathrm{mg}$ bimensual por vía subcutánea) y leuprolide (22,5 mg trimestral por vía intramuscular).

3. Antiandrógenos. Este grupo de fármacos se divide a su vez en 2 subtipos:

A. Esteroideos: Los antiandrógenos esteroideos son el acetato de ciproterona y acetato de megestrol, cuyo uso en primera línea de hormonoterapia no está recomendado ${ }^{8,11}$, debido a sus efectos adversos importantes como aumento de peso, retención hidrosalina, trombosis venosas profundas, etc.
B. No esteroideos: Estos fármacos producen un bloqueo competitivo de la unión de la dihidrotestoesterona con su receptor intracitoplasmático. Este grupo incluye la flutamida, la bicalutamida y la nilutamida, que se administran por vía oral. Estos fármacos usados en monoterapia reducen los niveles intracelulares de los andrógenos, pero pueden aumentar los niveles séricos de testosterona, a causa de un incremento compensatorio de la LHRH ${ }^{12}$. Entre los efectos adversos de estos fármacos cabe destacar la mastodinia, ginecomastia y pérdida de la libido por la transformación periférica de los andrógenos en estrógenos; toxicidad hepática (flutamida y nilutamida), fibrosis pulmonar (nilutamida) y diarrea.

La bicalutamida tiene la ventaja de conservar la potencia sexual; por lo que los pacientes jóvenes, que deseen conservar dicha función, podrían ser tratados en primera línea con bicalutamida (dosis de 50-150 $\mathrm{mg} / \mathrm{día}$ ) en monoterapia hasta la progresión tumoral, momento el que deben ser rescatados con los análogos de LHRH ${ }^{3}$.

4. Otros: A) Antagonistas de la LHRH (abarelix): se trata de un polipéptido que inhibe directamente el receptor de LHRH, sin provocar el fenómeno de "flare" inicial. Sin embargo, su empleo en la práctica clínica se ha visto limitado por sus efectos secundarios (liberación de histamina) ${ }^{13}$; B) Estrógenos: a pesar de que este grupo de fármacos consiguen una eficacia similar a la orquiectomía ${ }^{14}$; sus efectos adversos severos, como el incremento de la mortalidad de causa cardiovascular, han llevado a su abandono en la práctica clínica.

Como conclusión, el tratamiento de primera línea del CPM hormonosensible es la castración quirúrgica (orquiectomía bilateral) o bien la castración médica mediante el empleo de los análogos de LHRH en monoterapia, asociado inicialmente a un antiandrógeno no esteroideo (bicalutamida). El antiandrógeno debe iniciarse una semana antes al empleo del análogo y mantenerse durante las 2-3 semanas de haberse iniciado aquél. En pacientes jóvenes que deseen conservar la potencia sexual se puede iniciar con bicalutamida en monoterapia, y cuando la enfermedad progrese res- 
catar al paciente con análogos de LHRH. El bloqueo andrógenico completo (BAC), que consiste en asociar un análogo y un antiandrógeno, ofrece solamente un escaso beneficio en la supervivencia a los 5 años, de $2-3 \%(0-5 \%)$, a expensas de una mayor toxicidad, según se deriva de varios metaanálisis publicados ${ }^{15,16}$.

\section{Cáncer de próstata metastático hormono-refractario}

Los CPM, tras una fase inicial de hormonosensiblidad en la que la enfermedad responde a la deprivación andrógenica, progresan a una segunda fase en la que las maniobras hormonales de segunda línea sólo consiguen una tasa de respuesta de 20$40 \%{ }^{17}$. Es conveniente asegurar que la enfermedad ha alcanzado esta fase de hormonorefractariedad mediante la determinación de los niveles séricos de testosterona, que ha de demostrar niveles propios de la castración. A falta de estudios concluyentes, se recomienda que una vez alcanzada esta fase se mantenga el tratamiento con los análogos de LHRH, teniendo en cuenta la tolerabilidad y otros factores individuales, como consecuencia de los resultados del estudio retrospectivo llevado a cabo por Taylor et $\mathrm{al}^{18}$. En dicho estudio se evaluó a 241 pacientes incluidos en 4 ensayos clínicos, demostrando un beneficio en la supervivencia global en los casos que continuaron con el bloqueo androgénico.

Cuando se alcanza esta fase de la enfermedad existen 2 modalidades terapéuticas:

A. Hormonoterapia de segunda línea: Ninguna maniobra hormonal de segunda línea ha demostrado aumentar la supervivencia global, y su papel es puramente paliativo. Está indicado en los casos asintomáticos con progresión bioquímica (elevación de PSA) o paucisintomáticos. La primera maniobra hormonal debe ser la retirada del antiandrógeno en caso de BAC. Con esta maniobra se consiguen una tasa de respuesta de $20-35 \%$ 19, 20 . La retirada del antiandrógeno consigue una mediana de duración de respuesta de 3-5 meses.

Existen otras muchas maniobras hormonales de segunda línea como el empleo de antiandrógenos, corticoides a dosis bajas y la inhibición de los andrógenos de las glándulas suprarrenales con el empleo de ketoconazol e hidrocortisona o aminoglutimide.
B. Quimioterapia paliativa: La quimioterapia paliativa en los casos de CPM hormono-refractarios está indicado si existe enfermedad sintomática o rápidamente progresiva. Hasta la aparición de los nuevos esquemas basados en docetaxel, la quimioterapia no había demostrado aumentar la supervivencia en el CPM.

Los agentes de quimioterapia empleados clásicamente con actividad en el CPM son la vinorelbina, mitoxantrone, cisplatino, estramustina o ciclofosfamida $^{21}$, que habían demostrado conseguir una estabilización de la enfermedad, con mejoría bioquímica y sintomática.

En la era pre-docetaxel la combinación de mitoxantrone a dosis de $12 \mathrm{mg} / \mathrm{m} 2 \mathrm{IV}$ día $1^{\circ}$ del ciclo y prednisona $5 \mathrm{mg} / 12$ horas VO cada 21 días, se convirtió en el estándar del tratamiento ${ }^{22}$. El estudio canadiense que incluía 161 pacientes con CPM hormono-refractarios demostró que dicha combinación era superior a la prednisona sola en términos de control sintomático, sin poder demostrar aumento en la supervivencia global. Un segundo estudio aleatorizado fase III que también contenía mitoxantrone, llevado a cabo por Kantoff et al, comparó la combinación de mitoxantrone $\left(14 \mathrm{mg} / \mathrm{m}^{2} \mathrm{IV}\right)$ e hidrocortisona frente a la hidrocortisona sola (40 $\mathrm{mg} /$ día VO) en 242 pacientes. Este estudio también demostró la superioridad de la combinación de mitoxantrone y el corticoide en el control de los síntomas, pero sin conseguir demostrar un beneficio en la supervivencia global ${ }^{23}$.

Otro agente quimioterápico a tener en cuenta en el control de los síntomas de los pacientes con CPM hormonorresistente es la vinorelbina. La vinorelbina es un alcaloide de la vinca, eficaz en el tratamiento de diversas neoplasias como el cáncer de pulmón no microcítico, cáncer de mama metastático o cáncer de cabeza y cuello. En un estudio aleatorizado fase III con 414 pacientes publicado en 2004 por Abratt et $\mathrm{al}^{24}$, en el que se comparaba la combinación de vinorelbina (30 mg/m $\mathrm{m}^{2}$ días 1 y 8 , cada 21 días) y hormonoterapia frente a la hormonoterapia sola (aminoglutimide e hidrocortisona 40mg/día), se demostró un beneficio clínico en la rama de la quimioterapia en el control de los síntomas (intensidad del dolor), así como una mayor tasa de respuestas bioquímicas (disminución de los niveles de PSA) y un mayor tiempo hasta la progresión de la enfermedad. 
TABLA I

Fármacos empleados en cáncer de próstata metastático (CPM) hormono-refractario

\begin{tabular}{|c|c|c|}
\hline Tipo de fármaco & Mecanismo de acción & Observaciones \\
\hline $\begin{array}{l}\text { Agentes clásicos: } \\
\text { Mitoxantrone, } \\
\text { Vinorelbina }\end{array}$ & - & $\begin{array}{l}\text { Útil en el manejo sintomático, pero no aumentan la supervivencia } \\
\text { Útil en pacientes con cardiopatía o mal estado general. }\end{array}$ \\
\hline $\begin{array}{l}\text { Taxanos: } \\
\text { Docetaxel }\end{array}$ & $\begin{array}{l}\text { Inhibición de la despolimerización } \\
\text { de microtúbulos }\end{array}$ & Aumenta de forma significativa la supervivencia global. \\
\hline $\begin{array}{l}\text { Antiangiogénicos: } \\
\text { Talidomida, } \\
\text { Bevacizumab, } \\
\text { Atrasertan }\end{array}$ & Inhibición de la angiogenésis tumoral & $\begin{array}{l}\text { En experimentación en el CPM. } \\
\text { Talidomida se emplea en mieloma refractario. } \\
\text { Bevacizumab se emplea en cáncer de colon metastático junto con } \\
\text { la quimioterapia. }\end{array}$ \\
\hline $\begin{array}{l}\text { Vacunas } \\
\text { antitumorales }\end{array}$ & Inmunoterapia génica & En experimentación en el CPM \\
\hline $\begin{array}{l}\text { Pro-apoptóticos: } \\
\text { Retinoides, } \\
\text { Oligonucleótidos } \\
\text { antisentido anti-Bcl-2 }\end{array}$ & $\begin{array}{l}\text { Promueven la muerte celular } \\
\text { programada (apoptosis) }\end{array}$ & $\begin{array}{l}\text { Los oligonucleótidos antisentido anti-Bcl-2(oblimersen) presentan } \\
\text { sinergismo con los taxanos, y producen reducción de los niveles } \\
\text { de Bcl-2 (potente inhibidor de la apoptosis). }\end{array}$ \\
\hline $\begin{array}{l}\text { Inhibidores de } \\
\text { proteosomas: } \\
\text { Bortezomib } \\
\text { (PS-341) }\end{array}$ & $\begin{array}{l}\text { Actividad inhibitoria del crecimiento } \\
\text { celular y citotoxicidad. }\end{array}$ & $\begin{array}{l}\text { En experimentación en CPM. } \\
\text { Botezomib se emplea en el mieloma múltiple refractario. }\end{array}$ \\
\hline
\end{tabular}

No obstante, dicha combinación no consiguió demostrar un aumento en la supervivencia global (la mediana de supervivencia fue de 15 meses).

Los 2 recientes estudios aleatorizados fase III, que comparan la combinaciones con docetaxel frente al esquema considerado estándar (mitoxantrone y prednisona), por primera vez consiguen demostrar un aumento en la supervivencia global, a expensas de una mayor toxicidad.

El estudio TAX-327 llevado a cabo por Tannock et $\mathrm{al}^{25}$, que incluía 1006 pacientes comparó en tres brazos las combinaciones de 1) docetaxel trisemanal $\left(75 \mathrm{mg} / \mathrm{m} 2 \mathrm{IV}\right.$ día $\left.1^{\circ}\right)$ y prednisona $(5 \mathrm{mg} / 12$ horas VO), 2) docetaxel semanal $\left(30 \mathrm{mg} / \mathrm{m}^{2} /\right.$ semanal IV) y prednisona, y por último 3) mitoxantrone (12 $\mathrm{mg} / \mathrm{m}^{2}$ día $1^{\circ}$ ) y prednisona. Este estudio demostró un aumento significativo de la supervivencia global en el brazo de docetaxel trisemanal (mediana de 18,9 meses) frente a los otros dos brazos (17,4 meses en la pauta semanal y 16,5 meses en el brazo de mitoxantrone). El control de los síntomas, especial- mente el dolor, fue superior en los brazos de docetaxel. Sin embargo, la toxicidad hematológica grado III-IV fue mucho mayor en el brazo de docetaxel trisemanal.

El estudio SWOG 99-16, llevado a cabo por Petrylak et $\mathrm{al}^{26}$, incluye a 770 pacientes que fueron aleatorizados a los dos brazos del estudio, que comparaba la combinación de docetaxel $\left(60 \mathrm{mg} / \mathrm{m}^{2} \mathrm{IV}\right.$ día $\left.2^{\circ}\right)$ y fosfato de estramustina $(280 \mathrm{mg} / 8$ horas VO días $1^{\circ}-5^{\circ}$ del ciclo), frente al esquema clásico de mitoxantrone y prednisona. En este estudio también se demostró un aumento significativo de la supervivencia global en el brazo del docetaxel, con un aumento de 1,9 meses en la mediana de supervivencia global a favor del docetaxel (17,5 meses vs 15,6 meses con un valor de $\mathrm{p}=0,02$ ). Así mismo, hubo en el brazo de docetaxel un aumento significativo de la tasa de respuesta bioquímica y de la supervivencia libre de progresión ( 6 meses vs 3 meses, con $\mathrm{p}<0,05)$.

Estos dos estudios han llevado a un cambio en el 
tratamiento estándar de los CPM hormono-refractarios. Sin embargo, los esquemas de quimioterapia con vinorelbina o mitoxantrone deben tenerse en cuenta en pacientes con un peor estado general o comorbilidad cardiovascular importante.

Otros fármacos útiles en el tratamiento del CPM hormono-refractario son los bifosfonatos intravenosos (zolendronato) $)^{27,28} \mathrm{o}$ el empleo de radiofármacos (Samaruim-153).

Se están investigando sobre la utilidad de otros grupos de fármacos (Tabla I) en el CPM hormo-refractarios, tales como los retinoides, oligonucleótidos antisentido anti-blc-2, imatinib, gefitinib, bortezomib, talidomida, vacunas anti-tumorales o análogos de vitamina $\mathrm{D}$.

\section{Bibliografía}

1. Calvo Manuel FA, Pérez Manga G. Tratamiento de la enfermedad localizada en Cáncer de Próstata, de Documentos Consensos Oncología. Ed Nova Sidonia. Madrid 2002.

2. Jemal A, Tiwari RC, Murria T et al. Cancer Statitics 2004. CA Cancer J Clin 2004;54:8-15.

3. Kasimis B, Wilding G, Kries W et al. Survival of patients who had salvage castration after failure on bicalutamide monotherapy for stage D2 prostate cancer. Can cer Invest 2000;18:602-608.

4. Davis NS, DiSant'Agnese PA, Ewing JF, Mooney RA. The neuroendocrine prostate: characterization and quantitation of calcitonin in the human prostate gland. J Urol 1989;142:884-888

5. Montie JE, Wood DP, Mendendorp SV, Levin HS, Pontes JE. The significance and management of transitional cell carcinoma of the prostate. Semin Urol 1990;7:262-268.

6. Adolfsson J. The natural history of early prostate cancer and the impact of endocrine treatment. Eur Urol 1999;36 Suppl 2: 3-8.

Correspondencia: Dr. Parham Khosravi Shahi Servicio de Oncología Médica HGU Gregorio Marañón Doctor Esquerdo, 46 E-28007 Madrid drkhosravi@hotmail.com
7. Civantos F, Marcial MA, Banks ER et al. Pathology of androgen deprivation therapy in prostate carcinoma. A comparative study of 173 patients. Cancer 1995;75:1634-41.

8. Loblaw A, Mendelson DS, Talcott JA et al. American Society of Clinical Oncology recommendations for the initial hormonal management of Androgen-sensitive metastatic, recurrent, or progressive prostate cancer. J Clin Oncol 2004;22:2927-41.

9. Byar DP, Crole DK. Hormone therapy for prostate cancer. Results of the Veterans Administration Cooperative Urological Research Group Study. Natl Cancer Inst Monogr 1998;7:165-70.

10. Pilepich MV, Caplan R, Byhart RW et al. Phase III trial of androgen supression using goserelin in unfavourable-prognosis carcinoma of the prostate treated with definitive radiotherapy: report of the Radiation Therapy Oncology Group Protocol 85-31. J Clin Oncol 1997;15:1013-21.

11. Bonomi P, Pessis D, Bunting N, et al. Megestrol acetate used as primary hormonal therapy in stage D prostatic cancer. Semin Oncol 1985;12:36-39.

12. Migliari R, Balzano S, Scarpa RM. Short-term effects of flutamide administration on hypothalamic-pituitary-testicular axis in men. J Urol 1998;139:637-9.

13. Mc Leod D, Zinner N, Tomera K et al. A phase III multicenter open-labed randomized study of abarelix versus leuprolide acetate in men with prostate cancer. Urology 2001;58:756-61.

14. Cox RL, Crawford ED. Estrogens in the treatment of prostate cancer. J Urol 1995;154:1991-8.

15. Schmitt B, Wilt TJ, Schellhammer PF et al.Combined androgen blockade with nonsteroidal antiandrogens for advanced prostate cancer: a systematic review. Urology 2001;57:727-732. 
16. Samson DJ, Seidenfeld J, Schmitt B et al. Systematic review and meta-analysis of monotherapy compared with combined androgen blockade for patients with advanced prostate carcinoma. Cancer 2002;95:361-76.

17. Kojima S, Suzuki H, Akakura et al. Alternative antiandrogens to treta prostate cancer relapse after initial hormone therapy. J Urol 2004;171:679-83.

18. Taylor CD, Elson P, Trump DL. Importance of continued testicular supression in hormone-refractory prostate cancer. J Clin Oncol 1993;11:2167-72.

19. Small EJ, Srinivas S. The antiandrogen withdrawal syndrome. Experience in a large cohort of unselected patients with advanced prostate cancer. Cancer 1995;76:1428-33.

20. Dawson NA, Mc Leod DG. Dramatic prostate specific antigen decrease in response to discontinuation of megestrol acetate in advanced prostate cancer: expansion of the antiandrogen withdrawal syndrome. J Urol 1995;153:194653.

21. Yagoda A, Petrilak D. Cytotoxic chemotherapy for advanced hormone-resistent prostate cancer. Cancer 1993;71:1098-109.

22. Tannock IF, Osoba D, Stockler MR et al. Chemotherapy with mitoxantrone plus prednisone or prednisone alone for symptomatic hormone-resistant prostate cancer: A canadian randomized trial with palliative end points. J Clin Oncol 1996;14:1756-64.

23. Kantoff PW, Halabai S, Conaway M et al. Hydrocortisone with or without mitoxantrone in Men with hormone-refractory Prostate Cancer: Results of the Cancer and Leukemia Group B 9182 Study. J Clin Oncol 1999;17:2506-13.

24. Abratt RP, Brune D, Melletios-Athanassios D et al. Randomized phase III study of IV vinorelbine plus hormonotherapy versus hormonotherapy alone in hormonorefractory prostate cancer. An Oncol 2004;15:1613-21.

25. Tannock IF, De Witt R, Berry W et al. Docetaxel plus prednisone or mitoxantrone plus prednisone for advanced prostate cancer. N Engl J Med 2004; 651: 1502-12.

26. Petrylak DP, Tangen C, Hussain M et al. Docetaxel and estramustine compared with mitoxantrone and prednisone for advanced refractory prostate cancer. N Engl J Med 2004; 651:1513-20.

27. Khosravi Shahi P, Díaz VM. Bifosfonatos en oncología. An Med Interna 2005; 22 (en imprenta).

28. Hernández JL, Riancho JA, González J. Bifosfonatos intravenosos. Med Clin (Barc) 2005;124:384-54 . 